Théologiques

Théologiques

\title{
Les Églises de l'Orient arabe face à la mondialisation du dialogue islamo-chrétien
}

\section{Fadi Daou}

Volume 19, numéro 2, 2011

Le dialogue islamo-chrétien

URI : https://id.erudit.org/iderudit/1024729ar

DOI : https://doi.org/10.7202/1024729ar

Aller au sommaire du numéro

\section{Éditeur(s)}

Faculté de théologie et de sciences des religions, Université de Montréal

ISSN

1188-7109 (imprimé)

1492-1413 (numérique)

Découvrir la revue

Citer cet article

Daou, F. (2011). Les Églises de l'Orient arabe face à la mondialisation du dialogue islamo-chrétien. Théologiques, 19(2), 89-99.

https://doi.org/10.7202/1024729ar
Résumé de l'article

Cet article représente une étude comparative des programmes du dialogue interreligieux pour les musulmans et les chrétiens de l'Orient arabe. L'auteur défend la thèse que la période qui suit les attaques terroristes du 11 septembre 2001 voit une évolution majeure sur le plan du dialogue et pose de nouveaux défis pour ses protagonistes. L'entrée en force des grandes institutions politiques musulmanes, sur la scène du dialogue, le propulse en effet sur le plan mondial et inter-civilisationnel, sans pour autant résoudre ses enjeux locaux. Cette situation crée un décalage entre les attentes des chrétiens d'Orient face au dialogue - dialogue qui est vital pour leur présence et le maintien du " vivre ensemble » dans leurs sociétés - et les attentes des musulmans qui cherchent à corriger par le dialogue l'image de l'islam qui est projetée mondialement. Ce décalage représente un enjeu, voire un risque pour l'avenir du dialogue au Moyen-Orient. Ce risque peut cependant être transformé en une circonstance favorable, suivant les hypothèses conclusives de l'auteur. 


\title{
Les Églises de l'Orient arabe face à la mondialisation du dialogue islamo-chrétien
}

\author{
Fadi DAOU* \\ Théologie politique \\ Université Saint-Esprit (Beyrouth, Liban)
}

Le climat des relations entre l'islam et l'Occident a indubitablement été affecté par les attentats du 11 septembre 2001. Depuis, nous sommes entrés dans une nouvelle phase dans le domaine du dialogue interreligieux. Une mondialisation du dialogue s'affirme, avec de nouvelles initiatives dont des personnes et des institutions musulmanes sont à l'origine. Cette nouvelle approche vient donc s'ajouter à une dynamique riche de son histoire et de la variété des expériences qui la composent. Le dialogue islamochrétien couvre, en effet, un grand nombre d'initiatives locales ou internationales, privées ou institutionnelles, qui ont déjà produit un certain nombre de fruits dans la purification des mémoires ou le dépassement des préjugés, ainsi que le développement d'espaces de solidarité intercommunautaire. Le chemin à parcourir ensemble reste toutefois long et les moyens utilisés pour avancer dans cette aventure sont toujours à actualiser ou à réinventer, en fonction des contextes différents et des nouveaux défis.

Ainsi, si le dialogue sur le plan mondial semble s'imposer, pour faire face à une forme de généralisation de la confrontation, on se demande si cette approche peut ignorer, marginaliser ou remplacer les dynamiques locales de dialogue. S'il est souhaitable de relever et de promouvoir l'aspect

* Fadi Daou est professeur du dialogue interreligieux et de théologie politique à l'Université Saint-Esprit (Liban) et président de la Fondation Adyan pour les études interreligieuses et la solidarité spirituelle. Ses recherches actuelles portent sur la théologie de la vérité publique. Il a récemment publié, en collaboration avec Nayla Tabbara (2013), L'hospitalité divine: l'autre dans le dialogue des théologies chrétienne et musulmane, LIT. 
positif de cette approche, on ne peut se passer d'un examen critique de son rapport à la réalité du dialogue et à ses enjeux, dans le domaine du «vivre ensemble» entre les communautés dans leurs différents contextes et leurs situations. La présente étude représente donc une brève analyse de la divergence entre certains programmes, au sein du dialogue qui a été prôné mondialement, ces dernières années, notamment par le roi Abdallah d'Arabie Saoudite et le prince Ghazi de Jordanie, d'un côté, et l'expérience séculaire et locale du dialogue selon les Églises de l'Orient arabe, de l'autre. Après la présentation de ces deux lignes d'action, je chercherai à préciser les enjeux d'une conciliation des deux qui pourrait faire appel à de nouvelles formes de dialogue.

\section{Les initiatives musulmanes du dialogue et leur ligne d'action, après le 11 septembre 2001}

Il est nécessaire d'interpréter les récentes initiatives du dialogue interreligieux, en général, et islamo-chrétien, en particulier, dans le cadre du nouveau contexte mondial, après les attentats aux États-Unis du 11 septembre $2001^{1}$. Sur le plan médiatique et culturel, ce contexte est déterminé par le développement de sentiments à la fois proches et antagonistes, dans les

1. Après le 11 septembre 2001, le contexte mondial, vu du Moyen-Orient, est marqué par une confrontation entre deux camps: d'un côté, le camp américain et, par extension, occidental, qui mène sa guerre préventive contre le terrorisme, loin de ses propres frontières, notamment en Afghanistan et en Irak, avec un appui relatif de la part de certains pays de la région; de l'autre, ce qui s'appelle l'axe de résistance antioccidental (mihwar al-mumanaa) qui est multiforme. Cet axe a en effet une dimension politique qui est portée par des États comme l'Iran principalement, mais aussi pragmatique, comme dans le cas de la Syrie. Ce même axe a, en deuxième lieu, une dimension militante qui constitue une opposition politique anti-occidentale à l'intérieur des régimes nationaux. Elle peut prendre une allure militaire, quand il s'agit de résister ou de combattre le premier axe ou ses alliés, comme pour le Hizbullah (Liban) et le Hamas (Palestine) - qui se considèrent comme une résistance militaire face à Israël - et certaines milices irakiennes ou afghanes qui combattent à la fois contre les forces américaines et leurs alliés occidentaux ou nationaux. Il se trouve certes des liens divers et complexes entre ces deux dimensions de l'axe de résistance anti-occidental. Il reste une troisième forme de cet axe qui est radicalement différente des deux premières; elle regroupe la nébuleuse des groupes jihadistes qui prônent le combat radical par des moyens terroristes contre l'Occident et ses alliés, y compris musulmans. Des attentats du 11 septembre 2001 aux États-Unis, de Madrid le 11 mars 2004 et de Londres le 7 juillet 2005, aux attentats quasi-quotidiens au Pakistan ou en Irak, contre les institutions politiques et militaires de ces pays ou contre des civils, il y a la même trace de la folie d'une violence absolue, qui cherche 
sociétés à majorité musulmane et en Occident. Les musulmans souffrent de la représentation déformée de leur religion, véhiculée d'un côté par les terroristes islamistes et de l'autre par l'islamophobie prépondérante, notamment dans les médias en Occident. Ils se considèrent également victimes d'une injustice internationale, liée à la pratique de deux poids deux mesures, en ce qui concerne les résolutions onusiennes non appliquées dans la cause palestinienne et les politiques occidentales en faveur d'Israël. Dans les sociétés occidentales, en revanche, on trouve les traces du traumatisme généré par les actes terroristes, qui alimentent l'islamophobie et qui mélangent le refus absolu de la violence au nom d'une appartenance religieuse avec la difficulté de comprendre et d'accepter la présence de communautés musulmanes immigrées en Occident représentant des phénomènes religieux atypiques et non-sécularisés.

C'est dans ce contexte-là que sont nées les grandes initiatives de dialogue provenant du côté musulman. Le congrès annuel de Doha, commandité par l'émirat du Qatar, a commencé à regrouper des représentants des différentes religions abrahamiques et autres, à partir de 2003, culminant avec l'inauguration du Centre de Doha pour le dialogue interreligieux $(\mathrm{DICID})^{2}$, en mai 2008. Le Centre se donne comme mission de contribuer au développement du dialogue entre croyants de différentes religions pour une meilleure compréhension des valeurs et des principes religieux et une collaboration au service de l'ensemble de l'humanité et de ses causes communes. La publication du Message d'Amman a pour sa part eu lieu dans le cadre des célébrations de Ramadan, le 9 novembre 2004, constituant un puissant appel et une invitation à la compréhension de l'islam authentique et au dialogue. La lettre Parole commune (PC), connue sous le nom de Lettre des 138 - le nombre de ses signataires, parmi lesquels se trouvent de grands dignitaires musulmans —, publiée le 13 octobre 2007, à l'adresse des chefs des Églises et des communautés chrétiennes, s'inscrit dans la même ligne que le Message d'Amman, autant dans son origine que dans sa forme littéraire. S'ajoute à ces initiatives l'Appel de la Mecque intitulé: L'islam et le dialogue interreligieux, constituant le message final du congrès islamique mondial, publié le 6 juin 2008. En application des décisions de ce congrès, le roi Abdallah d'Arabie saoudite a invité des représentants des religions du monde au Congrès international du dialogue interreligieux à

à déstabiliser l'axe ennemi, puisqu'il est impossible de le combattre et de le vaincre dans une guerre au sens conventionnel du terme.

2. Voir le site officiel du Centre sur: <http://www.dicid.org/>. 
Madrid, en juillet 2008, et il a demandé la tenue de l'assemblée générale des Nations Unies, en novembre 2008, sur les thèmes du dialogue interreligieux et de la promotion de la paix. Ces initiatives ne sont pas les seules, sans doute, ni les premières en matière de dialogue interreligieux, venant d'institutions ou de personnes musulmanes. On peut citer, par exemple, l'Institut royal d'études interreligieuses (RIIFS), fondé en Jordanie par le prince El-Hassan Ben Talal, en $1994^{3}$. Il faut toutefois remarquer que les nouvelles initiatives citées ci-dessus ont occupé diversement la place, tout en imposant ensemble un nouveau paradigme musulman du dialogue islamo-chrétien, ayant son propre programme.

Le Message d'Amman avec la Lettre des 138, d'une part, et l' "Appel de la Mecque ", d'autre part, nous fournissent en effet un plan assez clair de la nouvelle ligne d'action musulmane pour le dialogue interreligieux en général et le dialogue islamo-chrétien en particulier. Je le résume en cinq points :

1) Faire face aux préjugés, attaques et accusations injustes concernant l'islam dans ses aspects religieux, culturels ou politiques ${ }^{4}$.

2) Présenter l'islam dans ses fondements théologiques et ses valeurs authentiques, dans un but externe de témoignage, afin de corriger les perceptions que les autres en ont, et dans un but interne, pour une éducation religieuse correcte, purifiée des influences obscurantistes et adaptée aux exigences du monde contemporain ${ }^{5}$.

3) Faire une place à l'islam dans les débats et les initiatives concernant les problématiques civilisationnelles d'envergure mondiale, comme la paix ou les questions écologiques ${ }^{6}$.

4) Faire face aux courants ou mouvements terroristes qui agissent au nom de l'islam ou d'autres religions ou idéologies?.

5) Montrer que le dialogue est une exigence de foi, dans la religion musulmane, qui est la religion du juste milieu, et concrétiser cela par des

3. Voir le site officiel de l'Institut sur: <http://www.riifs.org/>.

4. Voir Message d'Amman (MA), $\mathbb{\$} 1$; "Appel de la Mecque» (AM), Objectifs du dialogue, 2 et 4.

5. Voir MA, tout le texte porte ce souci, notamment $\$ 11-12$; AM, objectif 1 ; Parole commune (PC).

6. Voir MA, $\mathbb{} 2$ et 11 ; AM, Objectifs 3 et 8 et Fondements du dialogue 2; PC, introduction et conclusion.

7. Voir MA, $\$ 10 ;$ AM, Thèmes du dialogue, 2. 
initiatives pour la construction de la paix entre les peuples et dans les sociétés ${ }^{8}$.

\section{La ligne d'action des chrétiens d'Orient pour le dialogue avec les musulmans}

Les chrétiens de l'Orient arabe, appartenant à plusieurs Églises et traditions différentes ${ }^{9}$, partagent avec les musulmans de leurs pays plus d'un millénaire d'histoire et un cadre civilisationnel et culturel, celui de l'arabité. Pour eux, le dialogue islamo-chrétien est inséparable de leur vie quotidienne, voire de leur identité culturelle et spirituelle. Dans leur premier message commun, les patriarches catholiques d'Orient s'adressent à «leurs frères musulmans » en ces termes:

Notre convivialité au long des siècles représente malgré toutes les difficultés, le terrain solide sur lequel il nous revient d'établir notre action commune, présente et future, au service d'une société égalitaire et harmonieuse, où nul ne se sent, quel qu'il soit étranger ou rejeté. Nous puisons à un héritage unique de civilisation. Chacun de nous a contribué à le former selon son génie propre. Notre parenté de civilisation est notre patrimoine historique. Nous tenons à le sauvegarder, à le faire évoluer, à le réenraciner et à le réactiver, de manière à ce qu'il soit le fondement de notre convivialité et de notre entraide fraternelle. Les chrétiens d'Orient sont une partie inséparable de l'identité culturelle des musulmans. De même, les musulmans en Orient sont une partie inséparable de l'identité culturelle des chrétiens. De ce fait, nous sommes responsables les uns des autres devant Dieu et devant l'histoire ${ }^{10}$.

8. Voir MA, $\$ 3-7 ;$ AM, Méthode du dialogue, 1 et Moyens du dialogue; PC.

9. La diversité des chrétiens d'Orient se lit de différentes manières. De par leur patrimoine liturgique et culturel, nous pouvons identifier six traditions: syriaque, assyrochaldéenne, grecque, copte, arménienne et arabe. La répartition conventionnelle se fait aujourd'hui selon la communion de foi. Cinq groupes distincts sont ainsi identifiés: le premier est celui des Églises orientales orthodoxes, théologiquement nonchalcédoniennes: copte, arménienne et syriaque; le second est celui des Églises orthodoxes, c'est-à-dire les différents patriarcats orthodoxes chalcédoniens: Antioche, Jérusalem et Alexandrie; le troisième groupe est celui des Églises catholiques, en communion avec Rome: maronite, melkite, arménienne, syriaque, copte, chaldéenne et latine; le quatrième groupe est celui des Églises et communautés protestantes et évangéliques; le dernier groupe ne contient que l'Église assyrienne d'Orient, une Église théologiquement non éphésienne, connue jadis en tant que nestorienne.

10. Conseil des patriarches catholiques d'Orient, «Un temps décisif pour les Églises du Moyen-Orient. Message à l'occasion de leur $1^{\text {er }}$ symposium », Bkerké (Liban), 24 août 1991, publié dans Documentation catholique, n 2037 (1991), p. 940. 
D'après ce texte, il est clair que les relations entre les musulmans et les chrétiens, dans l'Orient arabe, ont une structure fondamentalement différente d'autres relations similaires dans des contextes où l'une ou l'autre communauté est issue de l'immigration ou étrangère à la culture du pays. Des quatre formes du dialogue, selon une typologie devenue conventionnelle ${ }^{11}$, «le dialogue de la vie» exprime au mieux l'expérience partagée, vécue au quotidien par les chrétiens et les musulmans des pays arabes. Cette forme de dialogue constitue, en effet, pour eux, le cadre naturel de partage de leurs joies et de leurs peines, ainsi que des soucis communs de la vie quotidienne. Les trois autres formes du dialogue, à savoir le dialogue des œuvres au service de la libération de l'homme, le dialogue théologique et le dialogue des expériences spirituelles, ne sont pas non plus étrangères au programme du dialogue des chrétiens d'Orient. Dans une lettre pastorale des patriarches catholiques d'Orient, consacrée à cette question et publiée en 1994, sous le titre Ensemble devant Dieu pour le bien de la personne et de la société12, on peut lire tout l'ordre du jour proposé par ses auteurs au programme permanent du dialogue.

De ce texte, nous pouvons tirer la ligne d'action suivante:

1) Faire face ensemble aux nouveaux courants de réveil religieux qui s'expriment en logique de fanatisme et de fondamentalisme.

2) Assumer et répondre à l'enjeu de la relation entre religion et politique, de manière à dépasser le confessionnalisme vers une citoyenneté ouverte à la participation égale de tous à la vie publique.

3) Faire évoluer l'éducation et la formation de l'opinion publique, dans les familles, les écoles et à travers les médias, afin qu'elle contribue au dépassement des préjugés réciproques et favorise une connaissance mutuelle toujours plus approfondie.

4) Examiner et faire évoluer le discours religieux, notamment dans ses présupposés théologiques sur la théologie de l'autre.

5) Promouvoir la solidarité spirituelle entre les chrétiens et les musulmans, en se présentant devant Dieu dans la prière ensemble, comme frères, et en considérant la présence d'autrui comme étant la voix de Dieu pour le croyant.

11. Voir: Conseil pontifical pour le dialogue interreligieux et Congrégation pour l'évangélisation des peuples, Dialogue et annonce. Réflexions et orientations concernant le dialogue interreligieux et l'annonce de l'Évangile, $\mathrm{n}^{\circ} 42$.

12. Bkerké (Liban), 1994. 
Ce programme exprime la voix des pasteurs catholiques d'Orient et leurs aspirations pour l'avenir du dialogue islamo-chrétien. Il n'est pas très différent des autres approches chrétiennes locales. En effet, les autres communautés chrétiennes, orthodoxes et protestantes, abordent la question du dialogue avec les musulmans principalement à travers la structure œcuménique du Conseil des Églises du Moyen-Orient (MECC). Ce Conseil fut à l'origine de la création, dans les années 1990, du Groupe arabe du dialogue islamo-chrétien (GADIC) ${ }^{13}$, qui a pris plus tard son autonomie par rapport au Conseil. Le GADIC est actif surtout sur le plan de l'élaboration et de la propagation des principes sociopolitiques du «vivre ensemble» et du respect mutuel entre les religions. Quant au MECC, il assure une présence chrétienne institutionnelle sur la scène publique et dans la société civile, avec une attention particulière aux questions de justice et des droits de l'homme. En tenant compte de ces quelques nuances, on peut considérer que le résumé de la lettre des patriarches catholiques d'Orient représente globalement l'essentiel du plan d'action des chrétiens d'Orient pour le dialogue avec les musulmans. L'accent est toutefois à mettre davantage sur les trois premiers points, sachant que certaines Églises ou communautés seraient plus ou moins réticentes quant à la théologie de l'autre et à la solidarité spirituelle.

\section{Les enjeux et l'opportunité d'un dialogue «glocal»}

Autant dans son programme que dans son plan d'action, le dialogue prôné par les musulmans après le 11 septembre 2001, a été «globalisé ». L'appel de la Mecque a donné lieu au congrès international de Madrid puis à l'assemblée spéciale des Nations Unies, avec une série de rencontres sous forme de congrès de moindre envergure ou de séminaires dans plusieurs villes occidentales. Il est à noter que cette dynamique n'a abouti à aucune activité dans la région du Moyen-Orient. Il est en revanche vrai que la lettre Parole commune a été adressée à tous les chefs des Églises chrétiennes et, par conséquent, aux patriarches des Églises orientales non-catholiques. Les auteurs auraient considéré que le fait de l'adresser au pape, évêque de Rome, englobait ipso facto toutes les Églises dans la communion catholique, y compris les Églises orientales. Leur critère pour le choix des interlocuteurs fut donc «dénominationnel» et non communautaire ou

13. Voir le site officiel du Groupe sur: <http://www.agmcd.org/>. 
contextuel. D'ailleurs, les réponses et les événements qui ont suivi la publication de cette lettre se trouvent concentrés en Occident. D'une part, les autorités catholiques locales n'ont pas été associées à la liste des institutions concernées. D'autre part, les autres autorités ecclésiales d'Orient ne se sont pas senties concernées ou particulièrement intéressées par la démarche. Ni les Églises ni le MECC n'ont réagi avec l'attention accordée au texte, en Occident, par le Vatican ou l'archevêque de Canterbury, par exemple. En effet, pour les chrétiens de l'Orient arabe, les musulmans n'ont pas à se justifier ou à expliquer l'islam, dans son authenticité, face à des déformations ou à des amalgames généralisés. Certains de ces chrétiens contribuent même à défendre l'islam contre l'islamophobie et ses différentes manifestations en Occident. Ils n'abandonnent pas pour autant leurs propres objectifs, qui ne peuvent être atteints par des déclarations sur les principes ou l'affirmation de bonnes intentions.

La divergence entre les deux programmes reste en effet flagrante. Il faut dire aussi qu'ils répondent à des attentes et des besoins à la fois divergents et réels. Un ressentiment et une méfiance réciproques existent bel et bien, entre les musulmans et les Occidentaux. Et les initiatives ayant ce problème dans leur plan d'action sont plus que nécessaires. Le forum islamo-catholique en est un exemple. Réunissant au Vatican une assemblée paritaire de soixante chefs religieux et universitaires chrétiens et musulmans, à la suite de la lettre Parole commune, en novembre 2008, le forum conclut entre autres sur l'importance de l'instauration d'un comité mixte permanent pour coordonner les réponses aux conflits et aux autres situations d'urgence. Or, une lecture attentive de la liste des participants à ce forum islamo-catholique montre clairement une configuration explicitement mondialisée des deux religions. La délégation musulmane est en effet fortement marquée par la présence de nombreux musulmans occidentaux de nationalité voire d'origine, alors que dans la délégation catholique il faut noter la présence de représentants du christianisme minoritaire, comme dans les pays du Golfe et au Pakistan ${ }^{14}$. On peut également remarquer la rareté de participants chrétiens du Proche-Orient, eux qui portent bien plus que d'autres, à la fois la richesse et la complexité des relations islamo-chrétiennes, à la fois dans l'histoire et dans le présent.

La question se pose alors: comment ce dialogue "globalisé » peut-il prévenir des conflits ou réagir à des urgences qui, souvent, sont de nature

14. Pour les listes des participants et un compte rendu exhaustif du forum, voir Gaudel (2008). 
ou du moins d'origine locale et contextuelle? Ce dialogue pourrait sans doute contribuer de différentes manières au développement d'une réflexion commune et partagée, sur certains thèmes interreligieux. Il est cependant évident que nous risquons de passer à côté de la réalité des personnes et des communautés directement concernées par le "vivre ensemble », si nous considérons que leur programme local est dépassé ou remplacé par des enjeux mondiaux, voire des débats abstraits. Dans l'intérêt de tous, il faut chercher la complémentarité entre ces deux lignes d'action et relever les contradictions possibles aussi. Car j'estime que c'est surtout derrière ces contradictions que se cachent les futurs enjeux du dialogue.

Les communautés chrétiennes, dans l'Orient arabe, représentent un exemple flagrant du risque potentiel qui se trouve derrière la divergence des deux programmes. Dans le cadre du dialogue local, ces communautés se considèrent et sont considérées par leurs partenaires musulmans comme partie intégrante de la réalité socioculturelle de la région et cherchent à relever le défi du "vivre ensemble» dans ses différents aspects. Or, dans le cadre du dialogue "globalisé », ces communautés chrétiennes deviennent des minorités comparables aux minorités musulmanes, en Occident. Elles sont définies primordialement par ce qui les différencie de leur entourage plutôt que par la culture et le destin qui leur sont communs (Déclaration commune, point 6, p. 10$)$.

Ainsi, suivre un programme "globalisé » du dialogue place les problématiques sur un plan civilisationnel, où des communautés locales, comme les Églises d'Orient, risquent d'être considérées comme un prolongement de l'autre civilisation, sur un territoire qui n'est pas vraiment le leur. Bien que ce nouveau plan de dialogue semble susciter de l'intérêt de part et d'autre, une évaluation de ces initiatives s'impose pour mesurer leur impact réel sur le règlement des problèmes identifiés. Cela est important pour vérifier non seulement la pertinence de son programme, mais aussi la convenance de la méthode adoptée dans le cadre de ces démarches.

Pour cette évaluation qui reste à faire, j'avance deux hypothèses. La première consiste à noter que la typologie classique du dialogue, formulée par le Vatican, au début des années 1990, ne couvre plus la réalité actuelle incluant les nouvelles initiatives d'après 2001. Les quatre types déjà formulés restent néanmoins d'actualité. Il faudrait y ajouter une cinquième forme de dialogue politique et «intercivilisationnel ». On remarque aussi que le dialogue des expériences spirituelles et le dialogue de la vie et des œuvres communes ne sont pas directement concernés par les initiatives musulmanes citées précédemment. Ces formes de dialogue continuent toutefois 
à trouver des protagonistes des deux côtés, chrétien et musulman, et continuent à se vivre dans le cadre d'autres initiatives, d'allure moins officielle ou institutionnelle.

Ma deuxième hypothèse concernant l'évaluation du dialogue appelle à une synergie entre les deux plans du dialogue, «global» et local. Je pense qu'il est bon, voire nécessaire, de penser à un lien entre ces deux plans, non pour en créer un troisième, différent, ou pour faire des compromis entre les deux approches, mais pour introduire l'apport de chaque plan dans l'autre. Une "glocalisation» apporterait ainsi plus de réalisme au dialogue, fondé sur les expériences concrètes des personnes et des communautés, plus de réalisme aux initiatives mondiales, d'une part, et, d'autre part, ouvrirait le dialogue local à d'autres champs culturels et d'autres horizons d'interaction et de solidarité méconnus par un contexte particulier. Le plan «global » introduit ainsi une dynamique d'interculturalité interne à chaque religion et assouplit par conséquent certaines crispations identitaires. Aussi, les Églises de l'Orient arabe se trouveraient-elles, dans cette logique de "glocalité ", partenaires de l'islam dans son programme mondial et contribuant à corriger son image dans le monde, d'une part, et partenaires de l'Occident et des institutions chrétiennes mondiales, représentant par leur plan d'action local un défi positif au plan d'action musulman mondial, d'autre part. Sinon, quel sens donner au dialogue mondial intercivilisationnel si, localement, les chrétiens et les musulmans n'arrivent pas à relever ensemble les défis d'une culture citoyenne partagée et d'une éducation au «vivre ensemble» faisant ensemble face au fondamentalisme et prônant la solidarité et la fraternité ?

\section{Références}

Le Message d'Amman, 9 novembre 2004: <http://www.ammanmessage.com>.

Parole commune, 13 octobre 2007: <http://ar.acommonword.com>.

La ligue islamique mondiale, L'appel de la Mecque, 6 juin 2008.

Conseil pontifical pour le dialogue interreligieux et CongréGation POUR L'ÉVANGÉLISATION Des Peuples, Dialogue et annonce. Réflexions et orientations concernant le dialogue interreligieux et l'annonce de l'Évangile, Vatican, 1991.

CONSEIL DES PATRIARChES CATHOLIQUeS D'ORIENT, Un temps décisif pour les Églises du Moyen-Orient. Message à l'occasion de leur $1^{\text {er }}$ symposium, Bkerké (Liban), 1991. 
(1994), Ensemble devant Dieu pour le bien de la personne et de la société, Bkerké (Liban).

Gaudel, J. M. (2008), «Rome: le "forum" islamo-catholique", Se comprendre, 8/10, p. 1-14.

\section{Résumé}

Cet article représente une étude comparative des programmes du dialogue interreligieux pour les musulmans et les chrétiens de l'Orient arabe. L'auteur défend la thèse que la période qui suit les attaques terroristes du 11 septembre 2001 voit une évolution majeure sur le plan du dialogue et pose de nouveaux défis pour ses protagonistes. L'entrée en force des grandes institutions politiques musulmanes, sur la scène du dialogue, le propulse en effet sur le plan mondial et inter-civilisationnel, sans pour autant résoudre ses enjeux locaux. Cette situation crée un décalage entre les attentes des chrétiens d'Orient face au dialogue - dialogue qui est vital pour leur présence et le maintien du «vivre ensemble» dans leurs sociétés —, et les attentes des musulmans qui cherchent à corriger par le dialogue l'image de l'islam qui est projetée mondialement. Ce décalage représente un enjeu, voire un risque pour l'avenir du dialogue au Moyen-Orient. Ce risque peut cependant être transformé en une circonstance favorable, suivant les hypothèses conclusives de l'auteur.

\section{Abstract}

This paper represents a comparative study of the agendas of interreligious dialogue for Muslims and Christians in the Arab East. The author argues that the period following the terrorist attacks of September 11, 2001 saw a major shift in the dialogue and poses new challenges for its protagonists. Indeed the dynamic involvement of important Muslim political institutions in dialogue propelled it on a global and inter-civilizational level without solving its local issues. This creates a gap between the expectations of Eastern Christians concerning the dialogue - a dialogue that is vital to their presence and living-together with Muslims in their societies - and those of Muslims who seek to globally correct through dialogue the image of Islam. This shift is a challenge, even a risk for the future of dialogue in the Middle East, but it nevertheless can be transformed into an opportunity according to the concluding hypothesis of the author. 